\title{
Experiments of periodic forcing of Saffman-Taylor fingers
}

\author{
M. Torralba, ${ }^{1}$ J. Ortín, ${ }^{1}$ A. Hernández-Machado, ${ }^{1}$ and E. Corvera Poiré ${ }^{1,2, *}$ \\ ${ }^{1}$ Departament ECM, Facultat de Física, Universitat de Barcelona, Diagonal 647, E-08028 Barcelona, Spain \\ ${ }^{2}$ Departamento de Física y Química Teórica, Facultad de Química, UNAM, Ciudad Universitaria, México D.F. 04510, Mexico
}

(Received 10 September 2007; published 14 March 2008)

\begin{abstract}
We report on an experimental study of long normal Saffman-Taylor fingers subject to periodic forcing. The sides of the finger develop a low amplitude, long wavelength instability. We discuss the finger response in stationary and nonstationary situations, as well as the dynamics towards the stationary states. The response frequency of the instability increases with forcing frequency at low forcing frequencies, while, remarkably, it becomes independent of forcing frequency at large forcing frequencies. This implies a process of wavelength selection. These observations are in good agreement with previous numerical results reported in [LedesmaAguilar et al., Phys. Rev. E 71, 016312 (2005)]. We also study the average value of the finger width, and its fluctuations, as a function of forcing frequency. The average finger width is always smaller than the width of the steady-state finger. Fluctuations have a nonmonotonic behavior with a maximum at a particular frequency.
\end{abstract}

DOI: 10.1103/PhysRevE.77.036207

PACS number(s): 89.75.Kd, 47.54. $-\mathrm{r}, 47.61 . J d, 47.56 .+\mathrm{r}$

\section{INTRODUCTION}

Frequency dependent flows of fluids in confined media can be found in several fields. Cleaning of filters [1] and modern techniques of secondary oil recovery [2] are two examples of it.

Many of these situations imply the presence of more than one fluid. In particular, the displacement of a viscous fluid by a less viscous fluid is susceptible of undergoing a SaffmanTaylor instability [3], in which fingers of the less viscous fluid penetrate the more viscous fluid. In this paper we report on an experimental study of the destabilization of SaffmanTaylor (ST) fingers in frequency-dependent situations.

ST fingers can be generated in a controlled way in a HeleShaw (HS) cell [4], a pair of glass plates parallel to each other that form an almost two-dimensional channel in which the flow takes place. When a viscous fluid in the cell is displaced by a less viscous one, the interface between the two fluids becomes unstable to the formation of fingers. After a dynamic process of finger competition, a steady state is reached in which a single finger (the ST finger) occupies the channel. Due to its relative simplicity, the ST instability in a HS cell has become an archetype of interfacial instabilities in two-phase flow [5-8].

In the absence of anisotropy ST fingers are always wider than half the channel width. These fingers are called normal, in order to differentiate them from narrow fingers obtained in the presence of anisotropy, called anomalous [9-11]. While lateral instabilities in anomalous fingers have been known for two decades [10,11], normal ST fingers were considered for a long time to have stable flat sides. Recently, however, there have been several observations of lateral fluctuations of normal ST fingers. Moore et al. have reported experiments of lateral fluctuations of the ST finger in wide channels [12]. Also Torralba et al. have shown experimentally that quenched noise induces a small-amplitude long-wavelength

\footnotetext{
*Author to whom correspondence should be addressed; eugenia.corvera@gmail.com
}

instability on the sides of normal ST fingers [13]. Fluctuations on the finger sides have a dominant wavelength, indicating that the system acts as a selective amplifier of static noise. A numerical integration of a phase field model [14] has made evident two different ways of inducing lateral instabilities on normal ST fingers. First, by forcing the finger with a time-dependent pressure gradient, superimposed upon the constant pressure gradient necessary to produce the steady-state finger [15]. Second, through the presence of spatial perturbations [16]. Numerically, it has been found that both spatial and temporal perturbations are able to destabilize the finger flat sides with a small amplitude and long wavelength instability.

Here we present an experimental study of the destabilization of a normal air-oil ST finger in a HS channel, subject to periodic forcing. The steady-state finger is generated by withdrawing the oil at constant average flow rate. The finger is then periodically forced by imposing a periodic pressure signal on the side of the air. We find that the finger responds by generating a wave on its tip that propagates towards the finger sides. There is a dynamic process and, in most cases, the instability reaches a stationary state with a wavelength that no longer changes in the course of the experiment.

The paper is organized as follows. The experimental setup and the protocol used to analyze the experimental data are described in Sec. II, together with a qualitative description of our observations. The experimental results are presented in Sec. III. As reference, we have introduced two quantities that have units of frequency in the unperturbed problem. We discuss the finger response in stationary and nonstationary situations as well as the dynamics towards the stationary states. We also study how the average value of the finger width and its fluctuations depend on forcing frequency, and the role of finger velocity. Finally in Sec. IV we discuss the results and draw the conclusions.

\section{EXPERIMENTAL SETUP, OBSERVATIONS, AND ANALYSIS}

\section{A. Experimental setup}

Experiments have been performed in a rectangular HS cell made of two glass plates, $19 \mathrm{~mm}$ thick, placed horizon- 


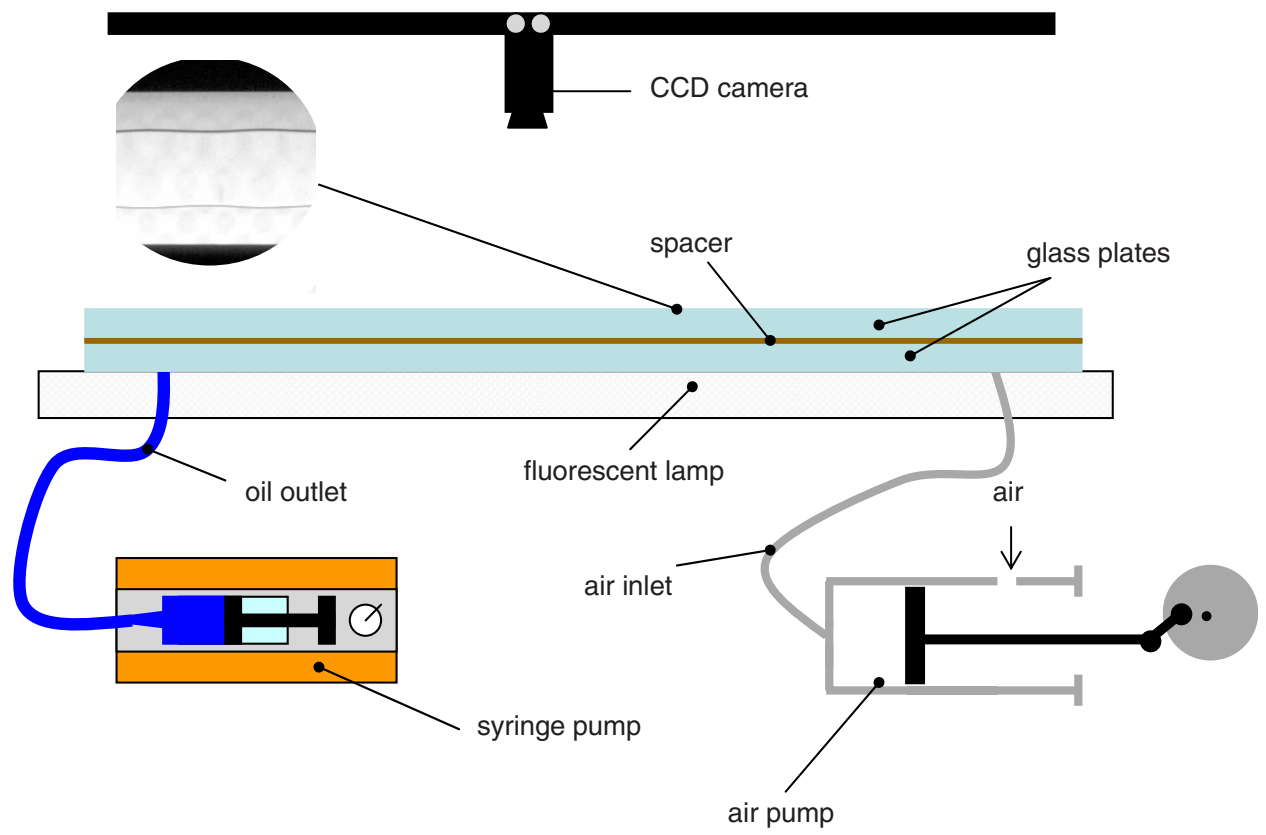

FIG. 1. (Color online) Sketch of the experimental setup.

tally one above the other, separated by a brass frame carefully machined to provide a gap spacing of $1.00 \mathrm{~mm}$, and firmly clamped to each other. A mechanical strain gauge with $20 \mu \mathrm{m}$ resolution, placed in contact with the top plate, does not reveal any measurable flexing of the plate in the course of the experiments. A sketch of the experimental setup is presented in Fig. 1. The bottom plate has a wide opening at each end. The distance between both openings is $L=1.15 \mathrm{~m}$. The channel provided by the brass frame has a width $W$ $=25.00 \mathrm{~mm}$. This gives an aspect ratio $L / W>40$, large enough to reach the wavelength selection regime reported in [15].

We have used a silicone oil (Rhodorsil 47V500) of dynamic viscosity $\eta=0.518 \pm 0.002 \mathrm{~Pa} \mathrm{~s}$ at $20^{\circ} \mathrm{C}$, density $\rho$ $=975 \pm 10 \mathrm{~kg} / \mathrm{m}^{3}$, and surface tension $\sigma=20.7 \mathrm{mN} / \mathrm{m}$, as viscous fluid. The oil has been colored with Oil Blue $\mathrm{N}$ (Sigma-Aldrich) without a measurable change in viscosity and surface tension, as verified by rheological and tensiometric tests.

The oil fills initially the cell. It is withdrawn from one end of the cell at constant average flow rate by means of a syringe pump. We selected flow rates of 36.0 and $71.0 \mathrm{~mm}^{3} / \mathrm{s}$. The corresponding withdrawal velocities (far away from the finger tip) are $V_{\infty}=1.42$ and $2.83 \mathrm{~mm} / \mathrm{s}$. At the opposite end of the cell the air inlet is connected to an air pump designed to produce a periodic oscillation of the air pressure around ambient pressure. The air pump consists of a mobile piston of $4400 \mathrm{~mm}^{2}$ of cross-sectional area, placed inside a plastic cylinder. The piston is able to oscillate at a frequency that can be set in the range $0-1 \mathrm{~Hz}$. The pump admits air from outside when the position of the piston is at one extreme. Initially the pump is off, and the piston is placed such that air may enter freely into the cell at ambient pressure. Quickly after fluid withdrawal starts, a finger of incoming air is formed. When this newly formed finger reaches a steady state (at a distance of approximately $6 \mathrm{~W}$ ) the air pump is switched on.

Figure 2 shows the pressure oscillations in the two fluids, recorded with pressure transducers placed in parallel to the fluid flow at the two ends of the cell. At the air inlet (bottom plot) the pressure perturbation is a periodic signal with a period formed of two parts: One period of a sinusoidal, to a very good approximation, and a constant signal. Both are produced by the oscillatory motion of the piston. The sinu-
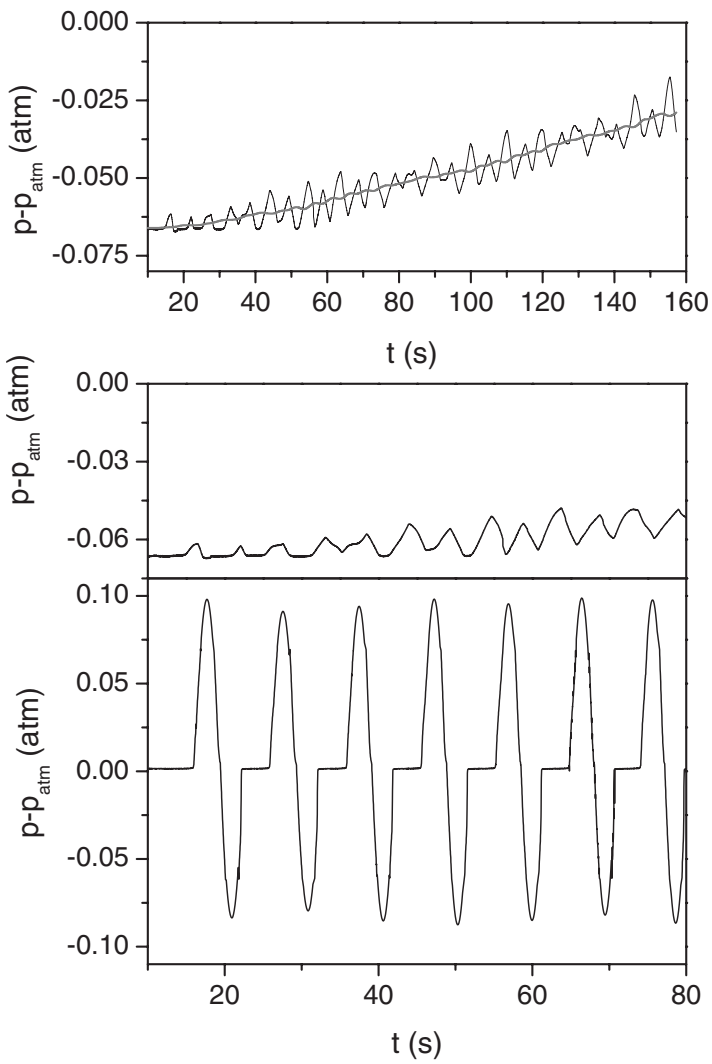

FIG. 2. Pressure modulations measured at the air inlet (bottom) and the oil outlet (middle and top), for an average flow rate of $36.0 \mathrm{~mm}^{3} / \mathrm{s}$ and a forcing frequency $\nu_{\text {in }}=0.11 \mathrm{~Hz}$. The ascending line on the top plot, drawn to guide the eye, is the average pressure signal obtained by smoothing out the actual oscillating signal. 
soidal part is produced when the piston moves ahead of the small hole that allows air to fill the pump. The constant part is produced when the piston is behind the small hole and therefore the pressure in the cell is essentially the atmospheric pressure. We have checked that the frequency of the pressure signal in the air follows exactly the frequency of forcing. At the oil outlet (middle plot) the pressure signal is still periodic. A frequency analysis shows that it contains the periodicity of the forcing and several other frequency components. The average pressure signal (obtained from smoothing out this oscillating pressure signal) takes its most negative value at the beginning, reflecting the suction of the syringe pump. As the finger tip advances in the cell at a constant average velocity, and approaches the oil outlet, this average pressure rises up to the atmospheric pressure linearly in time (top plot), because the pressure difference between the finger tip and the oil outlet becomes progressively smaller (the average pressure gradient remains constant and keeps the average tip velocity constant).

While experiments with ST fingers are usually controlled by a constant liquid flow rate or by a constant pressure difference, our forcing is a combination of the two. We control the average flow rate with the syringe pump, while the average input pressure is left free. But at the same time we control the pressure fluctuations with the air pump and let the flow rate fluctuate freely. This arrangement may seem complicated, but it is optimal for two reasons: (i) The controlled pressure fluctuations in the gas are transmitted nearly instantaneously to the finger tip, and (ii) the viscous pressure dissipation on the oil is so large that the flow rate fluctuations at the oil outlet are small compared to the average flow rate.

The cell is illuminated from below with a $35 \mathrm{kHz}$ fluorescent lamp, $1200 \mathrm{~mm}$ long, covered with white plastic to diffuse light. Images of the air finger are recorded by a CCD camera placed above the cell. The camera provides images of $640 \times 480$ pixels with a resolution of $0.16 \mathrm{~mm} /$ pixel. Since our cell is $25 \mathrm{~mm}$ wide, the region of interest captured in each image spans the whole cell width and a length of $102 \mathrm{~mm}$ along the finger corresponding approximately to $4 W$.

\section{B. Qualitative observations}

Once the periodic forcing is switched on, the finger tip responds with a periodic oscillation (a periodic backward and forward motion in the reference frame moving with the average velocity of the finger tip). This generates a wave that is born close to the tip and propagates towards the finger sides. The finger tip looks like the one of a normal ST finger, and the finger sides present a low-amplitude longwavelength instability reminiscent of incipient dendrites.

As the wave propagates away from the tip, the wavelength of the lateral instability undergoes a dynamic process. For most forcing frequencies the lateral modulations of the finger reach a stationary state, with a wavelength and amplitude that no longer change in time. When this happens, the shape of this part of the finger remains static in the laboratory frame of reference. However, there is an intermediate range of forcing frequencies (as discussed in the next sec-

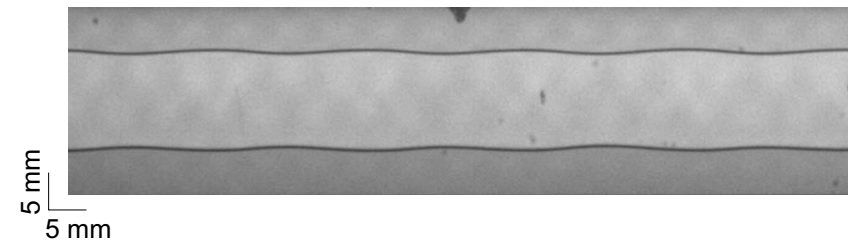

FIG. 3. Typical image recorded in our experiments. In this case the picture corresponds to a late stage of the dynamic process. Here the withdrawal velocity is $V_{\infty}=1.42 \mathrm{~mm} / \mathrm{s}$ and the forcing frequency $\nu_{\text {in }}=0.19 \mathrm{~Hz}$.

tion) for which a stationary state is never reached.

For forcing frequencies larger than $\nu_{\text {in }}=0.08$, we have checked that the shape of the wave in stationary states is nearly perfectly sinusoidal. An example of this wave is shown in Fig. 3. For $\nu_{\text {in }}<0.08 \mathrm{~Hz}$ the forcing is so slow that the wave follows the details of the forcing (which is periodic but not perfectly sinusoidal, as discussed above). The result is that the wave is not sinusoidal anymore. This is shown in Fig. 4.

\section{Data analysis}

\section{Regular data analysis}

Except for the lowest two forcing frequencies, discussed later, the recorded images have been analyzed in the following way.

The lateral modulations have been recorded as a function of time. To this purpose the camera has been fixed in the laboratory frame of reference and, as the fingertip advances towards the oil outlet, pictures in a fixed window of the HS cell have been taken. This window has been chosen following two criteria. First, the CCD camera must be placed far enough from the oil outlet to allow for the lateral instability of the finger to reach a stationary state. Second, to discard possible transient effects after switching on the periodic forcing, the window cannot be placed too close to the air inlet. With these two criteria in mind, the window has been set at $12.5 \mathrm{~W}$ of the air inlet. Image acquisition begins when the finger tip enters the window, and ends when the tip of the finger has travelled a distance of approximately $22 \mathrm{~W}$ beyond the window. A typical view of the recorded images (cropped to $640 \times 154$ pixels) is shown in Fig. 3 . We have acquired about 300 images per experiment for the low flow rate and about 150 images for the high flow rate. Out of these we

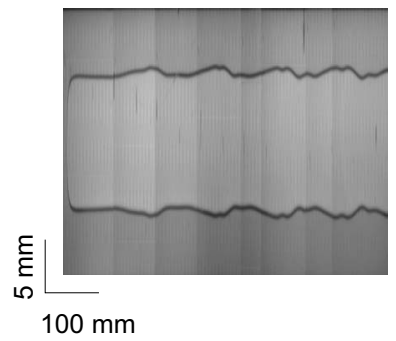

FIG. 4. Reconstructed image of a long finger corresponding to a withdrawal velocity $V_{\infty}=1.42 \mathrm{~mm} / \mathrm{s}$ and a forcing frequency $\nu_{\text {in }}$ $=0.03 \mathrm{~Hz}$. 
have analyzed one image every $T / 10 \mathrm{~s}, T$ being the total duration of an experiment.

The dynamic process undergone by the lateral modulations of the finger is visible in the time sequence of recorded images. When the wavelength of the lateral modulationmeasured as the distance between adjacent maxima or minima-no longer changes, we observe a saturation of the shape. Then, we compute the average wavelength of the modulations in one image, $\Lambda$, and take this value as the wavelength of the lateral instability for one realization of the experiment. In the same image we measure also the average finger width, $\lambda$. From these two measurements, a response frequency $\nu_{\text {out }}$ (the frequency that would be measured in the reference frame moving with the average velocity of the finger tip), for a given realization of the experiment, can be determined as

$$
\nu_{\text {out }}=\frac{U}{\Lambda}=\frac{V_{\infty}}{\lambda \Lambda},
$$

where $V_{\infty}$ is the velocity of the oil far away from the finger tip [17]. We have performed five independent realizations of the experiment for each $\nu_{\text {in }}$ studied. The final response frequency of the lateral instability - at a given $\nu_{\text {in }}$ is the weighted average of $\nu_{\text {out }}$ in the five realizations.

\section{Data analysis at low forcing frequency}

For the two lower values of $\nu_{\text {in }}$, the modulation of the finger sides is not sinusoidal. Instead, it presents a sawtooth profile, as shown in Fig. 4, which contains several frequencies. In addition, the largest wavelength of the profile is longer than the lengthwise span of our window of observation. The window cannot be made larger, because we would lose the resolution required to measure the amplitude of the instability. In general, since the wavelength evolves as it propagates far from the tip, an image reconstruction of the finger made of pictures taken at different times is not possible. However, for very low $\nu_{\text {in }}$ the structure that is born close to the fingertip remains unaltered in the laboratory frame of reference as the fingertip moves forward. We have thus decided to measure the lateral modulations in this kind of fingers by (i) letting the camera travel along the cell while it takes pictures of the finger, and (ii) making an image reconstruction of the whole finger by superposition of the recorded images. This technique was already used and described in [13].

In this case we have carried out three realizations of the experiment for each value of $\nu_{\text {in }}$. Each realization provides a reconstructed image of the whole finger. Each reconstructed image is then digitized, and a fast Fourier transform (FFT) is applied to repeated structures on one of the finger's sides. The position of the largest peak of the FFT is associated with the wavelength of the instability, $\Lambda$, for each realization. This, together with the mean finger width, $\lambda$, allows for the calculation of $\nu_{\text {out }}$. The final value of $\nu_{\text {out }}$ is the weighted average over the three realizations.

In order to check that this method gives reasonable results, we have applied both types of data analysis to the experiments at the lowest four $\nu_{\text {in }}$ for which the regular data analysis is possible. We have confirmed that the FFT analysis of the reconstructed image leads to the same values of $\nu_{\text {out }}$ than the regular analysis described at the beginning of this subsection.

\section{RESULTS}

In order to describe our experimental results in terms of characteristic magnitudes of the unperturbed ST problem, we introduce two quantities that have dimensions of frequency. The first one is determined by the flow very far from the finger tip, and can be defined as $\nu_{\infty}=V_{\infty} / W$. The second one is defined as $\nu_{\text {fing }}=U /(\lambda W)=V_{\infty} /\left(\lambda^{2} W\right)$, where $U$ is the finger velocity and $\lambda W$ is the finger width. The second equality comes from mass conservation in two dimensions [17].

In our experiments the forcing causes the width to oscillate. Hence, in the expressions above $\lambda W$ must be understood as the average finger width. Moreover, the average finger width is a function of $\nu_{\text {in }}$, as shown below. This means that, for given $W$ and $V_{\infty}$, there is no longer a single $\nu_{\text {fing }}$, but a band of frequencies characteristic of the finger, $\Delta \nu_{\text {fing }}$.

Next, we present our experimental results in terms of response frequencies, $\nu_{\text {out }}$, in order to make contact with the phase-field simulations reported in [15] and with our own work on lateral instabilities induced by quenched disorder [13]. It is also interesting to present the results in terms of measured wavelengths, and this is done at the end of this section.

\section{A. Stationary states}

Figure 5 presents the results for $\nu_{\text {out }}$, for a withdrawal velocity $V_{\infty}=1.42 \mathrm{~mm} / \mathrm{s}$. Open symbols correspond to stationary states.

Increasing zone. At low forcing frequency ( $\nu_{\text {in }}$ in the range $0-0.22 \mathrm{~Hz}$ ) the instability is already born with a wavelength very similar to the one exhibited by the finger when its shape saturates. This stationary state is reached at a distance $d_{\text {sat }} \simeq 2.5 \mathrm{~W}$ from the fingertip. A typical time sequence of the window of observation for a finger in this range of $\nu_{\text {in }}$ is shown in Fig. 6. As mentioned for $\nu_{\text {in }}>0.08 \mathrm{~Hz}$ the lateral modulation is very close to a sinusoidal. Note that the triangular-wave shape is an effect of the compression along one axis of the figure.

Our results for $\nu_{\text {out }}$ (Fig. 5) show that $\nu_{\text {out }}$ increases monotonically as a function of $\nu_{\text {in }}$, up to $\nu_{\text {in }} \simeq \nu_{\text {fing }}$. We will refer to this range of frequencies as increasing zone. Response frequencies in this zone seem to be bounded by $\nu_{\text {in }}$ and $\nu_{\text {in }} / 2$. At very low $\nu_{\text {in }}$ the response is linear. As $\nu_{\text {in }}$ increases, the response deviates from being linear, and the slope of $\nu_{\text {out }}$ versus $\nu_{\text {in }}$ decreases systematically.

Selection zone. For $\nu_{\text {in }} \geq 0.30 \mathrm{~Hz}$ the instability is born with a wavelength much smaller than the final one reached in the stationary state. In this case the finger shape saturates at $d_{\text {sat }} \simeq 14 W$. A typical time sequence of the window of observation in this range of $\nu_{\text {in }}$ is shown in Fig. 7. The amplitude of the instability is much smaller here than in the increasing zone, but the wavelength is still measurable following the procedure described in Sec. II C. 


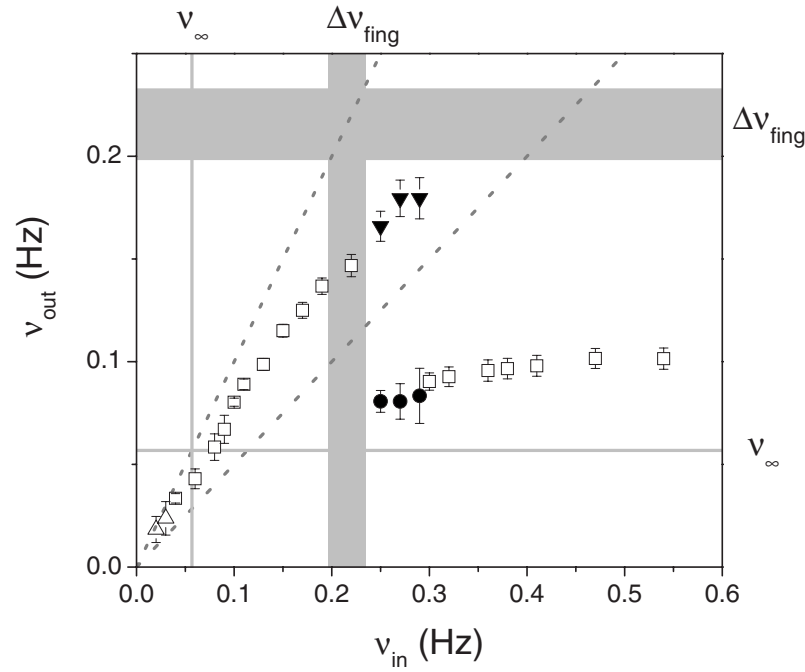

FIG. 5. Frequency of the lateral modulations, $\nu_{\text {out }}$, as a function of forcing frequency, $\nu_{\text {in }}$, for a withdrawal velocity $V_{\infty}$ $=1.42 \mathrm{~mm} / \mathrm{s}$. Measurements represented by the symbols $\square$ (stationary states) and $\boldsymbol{\nabla}$, (nonstationary states) have been obtained directly from the recorded images, as described in the text. The two measurements at low $\nu_{\text {in }}$ represented by $\Delta$ have been obtained from a FFT analysis of the image reconstruction. The error bars include data dispersion and instrumental uncertainty. For stationary states data dispersion is negligible for $\nu_{\text {in }}>0.1 \mathrm{~Hz}$. For nonstationary states, data dispersion is dominant. The dashed straight lines are guides to the eye, with slopes 1 and $1 / 2$. The frequency $\nu_{\infty}$ and the frequency band $\Delta \nu_{\text {fing }}$ are also represented, for reference. See text for details.

In Fig. 5 we observe that $\nu_{\text {out }}$ is almost independent of forcing frequency for $\nu_{\text {in }}>\nu_{\text {fing }}$ (i.e., at the right of the band $\Delta \nu_{\text {fing }}$ ). Consequently, we refer to this range of forcing frequencies as selection zone. The weighted average of $\nu_{\text {out }}$ in the selection zone, for $\nu_{\text {in }}$ in the range $0.30-0.54 \mathrm{~Hz}$ gives a selected frequency equal to $\nu_{\text {sel }}=0.096 \pm 0.002 \mathrm{~Hz}$. It is worth noting that $\nu_{\text {sel }}$ lies between the characteristic frequencies of the system, $\nu_{\infty}<\nu_{\text {sel }}<\nu_{\text {fing. }}$. See Table I.

Finally it is interesting to observe in Fig. 5 that, in the whole range of $\nu_{\text {in }}$ explored in the present experiments, $\nu_{\text {out }}$ always lies below the band $\Delta \nu_{\text {fing }}$, characteristic of the finger.

\section{B. Nonstationary states}

In a narrow frequency range between the increasing zone and the selection zone the instability does not reach a sta-

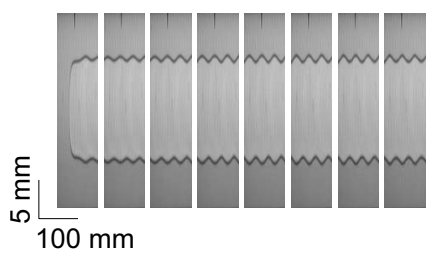

FIG. 6. Typical time sequence of recorded images for a finger in the increasing zone $\left(\nu_{\text {in }}=0.11 \mathrm{~Hz}\right.$ and $\left.V_{\infty}=1.42 \mathrm{~mm} / \mathrm{s}\right)$. Frames are separated $25 \mathrm{~s}$ (approximately $1 / 10$ of the total duration of the experiment).

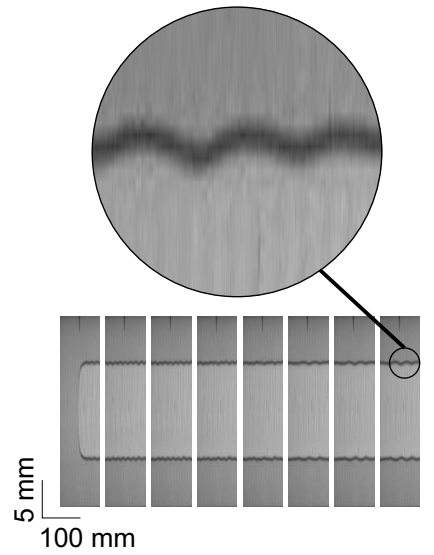

FIG. 7. Typical time sequence of recorded images for a finger in the selection zone $\left(\nu_{\text {in }}=0.36 \mathrm{~Hz}\right.$ and $\left.V_{\infty}=1.42 \mathrm{~mm} / \mathrm{s}\right)$. Frames are separated $25 \mathrm{~s}$ (approximately $1 / 10$ of the total duration of the experiment).

tionary state. At early and intermediate times the finger presents a sinusoidal response like the one observed in the increasing zone. Calculating $\nu_{\text {out }}$, by the usual procedure, for the last image that presents a sinusoidal profile in the time sequence, gives values of $\nu_{\text {out }}$ (the inverted black triangles in Fig. 5) that follow the trend of our previous results in the increasing zone. For some experimental realizations, however, we find that the sinusoidal profile of the fingers evolves into a slightly different pattern with a longer wavelength. This behavior is illustrated in Fig. 8. Although a stationary state is not reached, we have measured the wavelength of the last recorded frame and computed the corresponding value of $\nu_{\text {out }}$. The results, shown as black dots in Fig. 5, have a similar value than $\nu_{\text {sel }}$.

Hence the solid symbols in Fig. 5 indicate that, in this intermediate frequency range, a stationary state is not reached in the course of our experiment. For a number of experimental realizations, the response frequency $\nu_{\text {out }}$ of the lateral modulations seems to evolve from the value that would correspond to the increasing zone to the value of the selection zone.

\section{Average width and fluctuations of fingers}

We have computed the average finger width, $\lambda$, and the root mean square fluctuations of the finger width, $\delta_{\lambda}$, within the window of observation. The top plot in Fig. 9 shows that $\lambda$ depends on $\nu_{\text {in }}$ in a nonmonotonic way. We have also plotted the maximum and minimum finger width, $\lambda_{\max }$ and $\lambda_{\min }$. They are indicative of the amplitude of the lateral instability

TABLE I. Withdrawal velocities, range of values for the average finger width, characteristic frequency of the flow far from the finger tip, and frequency band of $\nu_{\text {fing }}$.

\begin{tabular}{lccc}
\hline \hline$V_{\infty}(\mathrm{mm} / \mathrm{s})$ & $\Delta \lambda$ & $\nu_{\infty}(\mathrm{Hz})$ & $\Delta \nu_{\text {fing }}(\mathrm{Hz})$ \\
\hline 1.42 & $0.498-0.535$ & 0.057 & $0.197-0.234$ \\
2.83 & $0.492-0.504$ & 0.114 & $0.449-0.470$ \\
\hline \hline
\end{tabular}




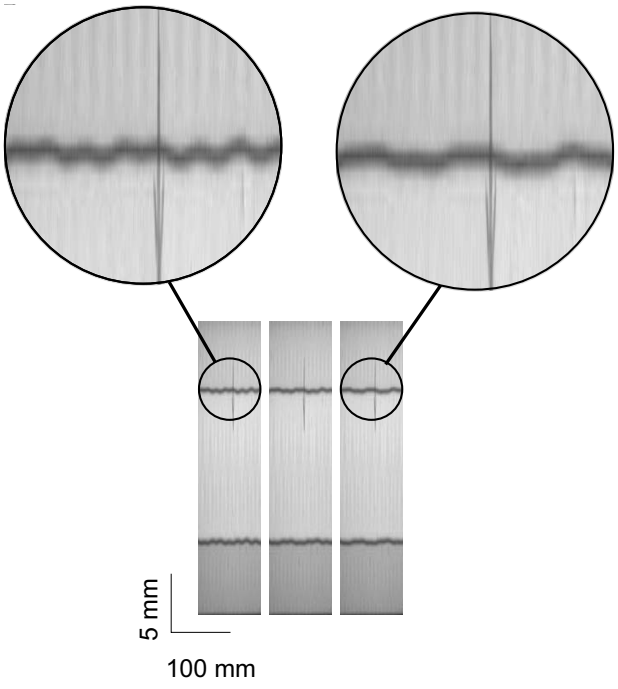

FIG. 8. Time sequence of images of the window of observation for a finger in a nonstationary state $\left(\nu_{\text {in }}=0.29 \mathrm{~Hz}\right.$ and $V_{\infty}$ $=1.42 \mathrm{~mm} / \mathrm{s}$ ). Frames are separated $25 \mathrm{~s}$ (approximately $1 / 10$ of the total duration of the experiment). The last frame displays lateral modulations of longer wavelength than the first frame.

at each frequency. The rms fluctuations of the finger width are shown in the bottom plot of Fig. 9. In the increasing zone $\left(\nu_{\text {in }}<\nu_{\text {fing }}\right)$ the rms fluctuations have a nonmonotonic behavior, with a pronounced maximum at $\nu_{\text {in }} \simeq \nu_{\infty}$. In the selection zone $\left(\nu_{\text {in }}>\nu_{\text {fing }}\right)$ the rms fluctuations are small and nearly independent of $\nu_{\text {in }}$.

In Fig. 9 we have also indicated the values of $\lambda$ and $\delta_{\lambda}$ measured in the absence of forcing $\left(\nu_{\text {in }}=0\right)$. Our results show that the average finger width $\lambda$ of the unperturbed ST finger

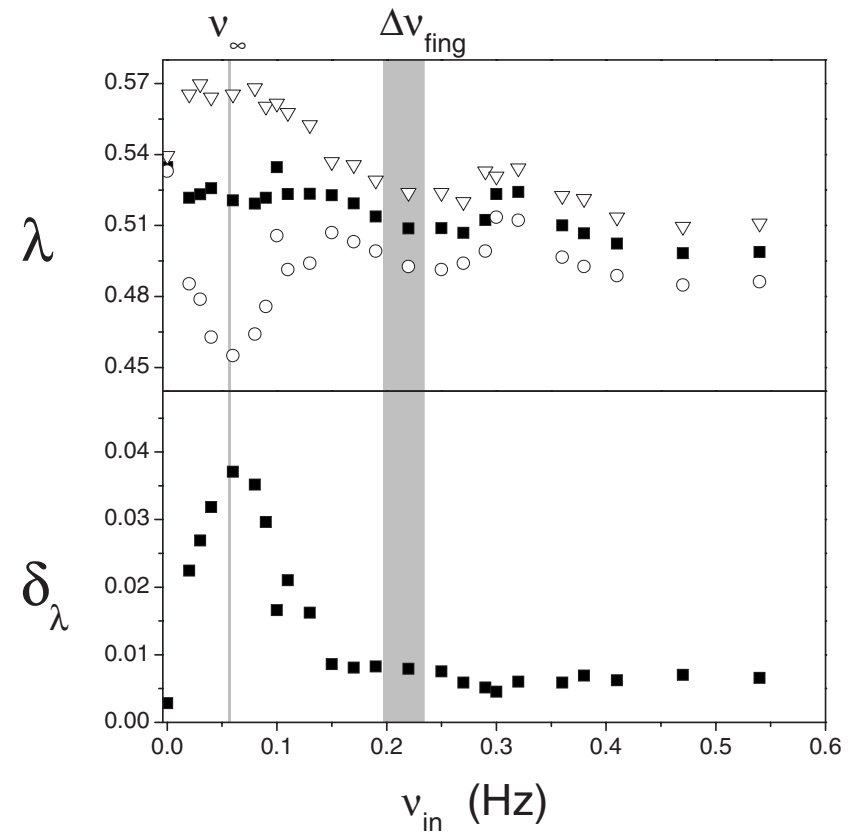

FIG. 9. Finger width, $\lambda$, and rms fluctuations of the finger width, $\delta_{\lambda}$, as a function of forcing frequency $\nu_{\text {in }}$, for $V_{\infty}=1.42 \mathrm{~mm} / \mathrm{s}$. Top: $\nabla, \lambda_{\max } ; \mathbf{\square}, \lambda$; and $\bigcirc, \lambda_{\min }$. Bottom: $\mathbf{\square}, \delta_{\lambda}$. The value of $\nu_{\infty}$ and the frequency band $\Delta \nu_{\text {fing }}$ are also shown for reference.



FIG. 10. Frequency of the lateral modulations, $\nu_{\text {out }}$, as a function of forcing frequency, $\nu_{\mathrm{in}}$. $\bigcirc$ : Stationary states for a withdrawal velocity $V_{\infty}=2.83 \mathrm{~mm} / \mathrm{s}$. The measurements have been obtained directly from the recorded images, as described in the text. The other symbols correspond to $V_{\infty}=1.42 \mathrm{~mm} / \mathrm{s}$, and are the same as in Fig. 5. The frequencies $\nu_{\infty}, \nu_{\infty}^{\prime}$, and the frequency bands $\Delta \nu_{\text {fing }}$ and $\Delta \nu_{\text {fing }}^{\prime}$ (the primed variables corresponding to the higher $V_{\infty}$ ) are also represented for reference.

is always larger than the average finger width of the forced ST finger. Conversely, the rms fluctuations of the width of the unperturbed ST finger are smaller than those of the forced fingers. This last result is indicative, first, of the experimental uncertainty of the steady-state finger width. And, second, it gives an estimate of the spatial resolution of our measurements. In other words, if for a given forcing frequency fluctuations were smaller than the ones measured for the steady state, we would not be able to detect them. Figure 9 shows that this is not the case, and thus the fluctuations reported can always be attributed to forcing.

\section{Dependence on finger velocity}

We have studied another withdrawal velocity, $V_{\infty}$ $=2.83 \mathrm{~mm} / \mathrm{s}$, in order to characterize the lateral instability of faster fingers. $V_{\infty}=2.83 \mathrm{~mm} / \mathrm{s}$ is a compromise value between two requirements: to have $V_{\infty}$ large enough for the dimensionless finger width to approach 0.5, and to have enough time in the course of the experiment to reach the steady state. At this higher velocity, both $\nu_{\infty}$ and the band $\Delta \nu_{\text {fing }}$ move towards higher frequencies, and the frequency range between $\nu_{\infty}$ and $\nu_{\text {fing }}$ widens. The characteristic frequencies for $V_{\infty}=2.83 \mathrm{~mm} / \mathrm{s}$ in Fig. 10 have been written with an apostrophe.

Figure 10 shows that the increasing zone at this higher velocity also extends up to frequencies of the order of $\nu_{\text {fing, }}$, as it was observed for the lower velocity. Interestingly, as long as $\nu_{\text {in }}$ is below the band $\Delta \nu_{\text {fing }}$, the response frequency $\nu_{\text {out }}$ is independent of $V_{\infty}$. To study the selection regime at this higher velocity, one would need to apply frequencies much higher than the ones accessible in our experiment. 


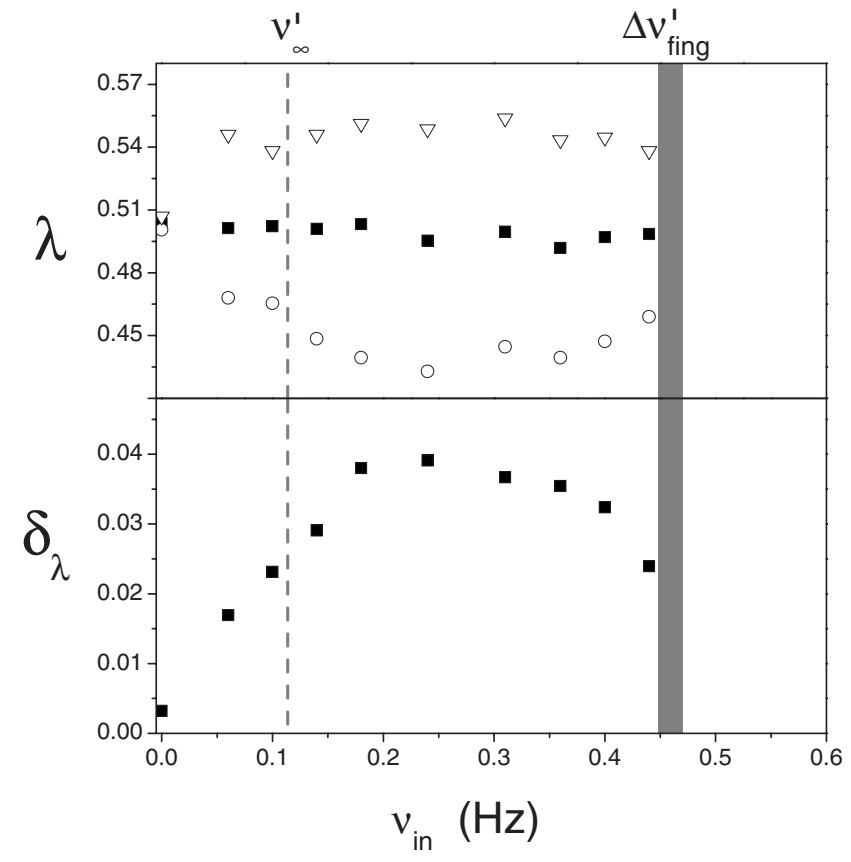

FIG. 11. Finger width, $\lambda$, and rms fluctuations of the finger width, $\delta_{\lambda}$, as a function of forcing frequency $\nu_{\text {in }}$, for $V_{\infty}$ $=2.83 \mathrm{~mm} / \mathrm{s}$. Top: $\nabla, \lambda_{\max } ; \boldsymbol{\square}, \lambda ; \bigcirc, \lambda_{\min }$. Bottom: $\boldsymbol{\square}, \delta_{\lambda}$. The value $\nu_{\infty}^{\prime}$ and the frequency band $\Delta \nu_{\text {fing }}^{\prime}$ are also shown for reference.

Results for the average finger width $\lambda$ and the rms fluctuations $\delta_{\lambda}$ at $V_{\infty}=2.83 \mathrm{~mm} / \mathrm{s}$ are shown in Fig. 11. The qualitative behavior of both magnitudes is very similar to the case $V_{\infty}=1.42 \mathrm{~mm} / \mathrm{s}$-in the frequency range from 0 to $\nu_{\text {fing }}$. At this higher velocity the average finger width is closer to the asymptotic value $1 / 2$, and remains nearly constant with $\nu_{\text {in }}$. The maximum of $\delta_{\lambda}$, on the other hand, is slightly shifted to $\nu_{\text {in }}>\nu_{\infty}^{\prime}$.

\section{E. Dimensionless wavelength of the lateral instability}

Figure 12 presents our results for the wavelength of the lateral instability $\Lambda$, rescaled by the finger width $\lambda W$, as a function of forcing frequency $\nu_{\text {in }}$, for the two withdrawal velocities $V_{\infty}=1.42$ and $2.83 \mathrm{~mm} / \mathrm{s}$.

The dimensionless wavelength decreases with $\nu_{\text {in }}$ but remains always larger than 1, i.e., the measured wavelength is always larger than the finger width. Actually, the end of the increasing zone is characterized by a dimensionless wavelength that approaches 1 .

At higher $\nu_{\text {in }}$ the lateral instability readjusts itself to a larger wavelength (for $V_{\infty}=1.42 \mathrm{~mm} / \mathrm{s}$ ). Notice that the finger width $\lambda$ decreases slightly with $\nu_{\text {in }}$ in the selection zone. Thus, upon rescaling the measured wavelength with $\lambda W$, we find that the resulting dimensionless wavelength is independent of $\nu_{\text {in }}$ in the selection zone.

Finally, we notice that the results in terms of wavelengths do depend on the withdrawal velocity $V_{\infty}$.

\section{DISCUSSION AND CONCLUSIONS}

Normal Saffman-Taylor fingers respond to a periodic pressure forcing by generating a wave on its tip. As the fin-

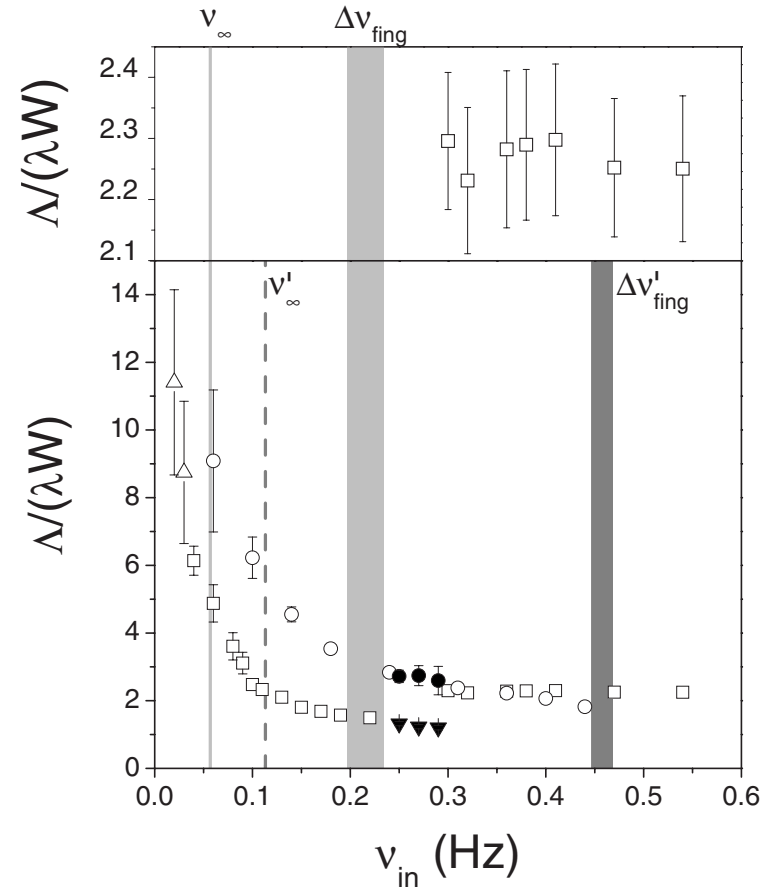

FIG. 12. Bottom: Wavelength of the lateral instability, $\Lambda$, rescaled by the finger width $\lambda W$, as a function of the forcing frequency $\nu_{\text {in }}$. The symbols $\triangle, \square, \nabla$, and correspond to $V_{\infty}=1.42 \mathrm{~mm} / \mathrm{s}$ and the same measurements as in Fig. 5. The symbol $\bigcirc$ corresponds to $V_{\infty}=2.83 \mathrm{~mm} / \mathrm{s}$ and the same measurements as in Fig. 10. The frequencies $\nu_{\infty}, \nu_{\infty}^{\prime}$, and the frequency bands $\Delta \nu_{\text {fing }}$ and $\Delta \nu_{\text {fing }}^{\prime}$ (the primed variables corresponding to the higher $V_{\infty}$ ) are also represented for reference. Top: Magnification of the selection zone for $V_{\infty}=1.42 \mathrm{~mm} / \mathrm{s}$.

ger advances, this wave propagates away from the tip towards the finger sides at constant velocity (in the finger frame of reference). For most forcing frequencies the amplitude and the shape of the wave saturate and give rise to a strictly periodic pattern on the sides of the finger.

These observations are in good qualitative agreement with the results of phase-field simulations reported in [15] in several frequency intervals. Thus, at very low forcing frequency the response is such that $\nu_{\text {out }}=\nu_{\text {in }}$, and the final frequency of the stationary state is reached near the finger tip. Also, at large forcing frequencies, a selection zone is reached where $\nu_{\text {out }}$ is almost independent of $\nu_{\text {in }}$ (although in the experiments it increases slightly with the forcing frequency), and falls between the two characteristic frequencies of the system, $\nu_{\infty}<\nu_{\text {out }}<\nu_{\text {fing. }}$. In this zone the stationary state is reached rather far from the finger tip. However, at intermediate forcing frequencies $\left(\nu_{\infty}<\nu_{\text {in }}<\nu_{\text {fing }}\right)$ the experimental observations differ from the numerical predictions. In the numerical integration of the phase-field model [15] $\nu_{\text {out }}=\nu_{\text {in }} / 2$ in this frequency range, the steady state has a frequency lower than the initial state, and the transition from the increasing zone to the transition zone is very abrupt. In experiments we find that $\nu_{\text {out }}$ increases with $\nu_{\text {in }}$, with a progressively decreasing slope between 1 and $1 / 2$, up to $\nu_{\text {fing }}$ (increasing zone). Furthermore, the lateral modulations reach a steady state at a short distance of the finger tip.

There are few forcing frequencies between the increasing zone and the selection zone for which a stationary state is 
never reached. For these forcing frequencies, the structures observed on the finger sides are roughly evolving from shapes characteristic of the increasing zone to shapes characteristic of the selection zone. It is possible that with a longer cell, a stationary state on the selection zone would have been reached. It is also possible that for these forcing frequencies two different response frequencies are equally likely, since we do not observe this shape evolution for all of the experimental realizations. Nevertheless, a thorough study of the non-stationary states falls beyond the scope of the present investigation.

The analysis of our results in terms of a dimensionless wavelength reveals that the average width of the finger, $\lambda W$, is a relevant length scale in the system. First, in the stationary state we do not observe wavelengths smaller than $\lambda W$. Thus, when the wavelength generated at the finger tip is shorter than the finger width, the system selects a much higher wavelength. It is also worth pointing out that the slight increase of $\nu_{\text {out }}$ observed in the selection zone is compensated by a slight decrease of the average finger width, resulting in a constant dimensionless wavelength $\Lambda / \lambda W$ in this zone.

We find that fingers subject to periodic forcing have an average finger width that depends on the forcing frequency in a nonmonotonic way. Moreover, we find that the average finger width is always smaller than the steady-state finger width. Also, we find that rms fluctuations of the finger width have a nonmonotonic behavior as a function of forcing frequency. At a certain forcing frequency, fluctuations are maximal. This maximum moves towards higher frequencies as the finger velocity increases.

ST fingers are known to become unstable when the modified capillary number $1 / B=12 \eta U / \sigma(W / b)^{2}$ becomes large. The tip-splitting instability has been studied by Park and Homsy [18] and by Tabeling et al. [19]. According to these authors tip-splitting takes place above $1 / B \simeq 3000$. In our experiments the finger is driven at moderate velocities, such that $1 / B \simeq 500$ or 1000 and, consequently, we do not have any observational evidence of tip-splitting.

It is interesting to compare the lateral modulations of normal ST fingers in our experiments with lateral modulations on anomalous ST fingers subject to forcing [11]. The anomalous fingers (narrower than half the channel width) are produced by some source of anisotropy in the system, for instance by a thread or two grooves. For anomalous fingers when periodic forcing is applied with an amplitude larger than the natural noise, there is side branching with strictly periodic undulations. In our experiments, where periodic forcing is applied, the wave of the stationary state is also strictly periodic. Interestingly, for anomalous fingers an optimal forcing frequency makes the amplitude of the lateral modulations maximal. In normal fingers we have been unable to observe the instability for low forcing amplitudes. For large frequencies, there is no instability observed in anomalous fingers. In our case, not only there is an instability at large frequencies but it presents the interesting regime of selection.

The present experimental study for forced fingers, our own experimental work on fingers in the presence of quenched disorder [13] and the numerical integration of the phase-field model for both, dynamic [15] and static [16] perturbations, lead to the conclusion that in normal SaffmanTaylor fingers lateral instabilities seem to be due to selective amplification of perturbations at the finger tip, just as it happens in the case of anomalous fingers [11] and in sidebranching in solidification [20,21].

From an applied point of view the presence of a lateral instability on normal ST fingers, driven with a timedependent pressure gradient, is relevant in designing secondary oil recovery procedures and medical applications that exploit the possibilities of periodic forcing. It has been argued that the amplitude of the instability might be very large at some particular (resonance) frequencies if the fluid displaced is viscoelastic [22]. A method for optimizing the displacement of a viscoelastic fluid has been reported on [23]. The importance of forcing has also been emphasized to enhance viscoelastic flow during occlusions [24]. The present study on Newtonian fluids provides a basis for future studies on the periodically forced displacement of complex fluids.

\section{ACKNOWLEDGMENTS}

We thank R. Ledesma-Aguilar and E. Alvarez-Lacalle for fruitful discussions. We gratefully acknowledge technical support from M. Quevedo and A. Comerma. We acknowledge financial support of the Dirección General de Investigación (MEC, Spain) under projects FIS2006-03525 and FIS2006-12253-C06-05, and of the catalan government under project 2005-SGR-00507. E.C.P. acknowledges the financial support from DGAPA, UNAM, through PAPIIT Grant No. IN101907 and from MEC, Spain, through Secretaría de Estado de Universidades e Investigación.
[1] See, for example, B. Ekberg and G. Norrgard, US Patent No. 4,946,602 (7 August 1990); A. R. Vadoothkey, US Patent No. 6,221,255 (24 April 2001); T Tuori, H. Sekki, J. Ihalainen, P. Pirkonen, H. Mursunen, US Patent No. 6,217,782 (17 April 2001); B. Ekberg, US Patent No. 5,900,147 (4 May 1999).

[2] See, for example, M. J. Economides, US Patent No. 5,460,223 (24 October 1995); C. Huh, P. L Wylie Jr., J. J. Shyeh, J. R. Bailey, US Patent No. 6,814,141 (9 November 2004); W. M. Harrison, US Patent No. 4,648,449 (10 March 1987); E. Cor- vera Poiré, M. López de Haro, and J. del Río Portilla, US Patent No. 7,201,224 (10 April 2007)

[3] P. G. Saffman and G. I. Taylor, Proc. R. Soc. London, Ser. A 245, 312 (1958).

[4] H. J. S. Hele-Shaw, Nature (London) 58, 34 (1898).

[5] P. Pelcé, Dynamics of Curved Fronts (Academic Press, Inc., San Diego, CA, 1988).

[6] K. V. McCloud and J. V. Maher, Phys. Rep. 260, 139 (1995).

[7] Y. Couder, in Perspectives in Fluid Dynamics, edited by G. K. 
Batchelor, H. K. Moffat, and M. G. Worster (Cambridge University Press, Cambridge, UK, 2000), p. 53.

[8] L. Ristroph, M. Thrasher, M. B. Mineev-Weinstein, and H. Swinney, Phys. Rev. E 74, 015201 (2006).

[9] A. T. Dorsey and O. Martin, Phys. Rev. A 35, 3989 (1987).

[10] Y. Couder, N. Gérard, and M. Rabaud, Phys. Rev. A 34, 5175 (1986).

[11] M. Rabaud, Y. Couder, and N. Gerard, Phys. Rev. A 37, 935 (1988).

[12] M. G. Moore, A. Juel, J. M. Burgess, W. D. McCormick, and H. L. Swinney, Phys. Rev. E 65, 030601(R) (2002).

[13] M. Torralba, J. Ortín, A. Hernández-Machado, and E. Corvera Poiré, Phys. Rev. E 73, 046302 (2006).

[14] A. Hernández-Machado, A. M. Lacasta, E. Mayoral, and E. Corvera Poiré, Phys. Rev. E 68, 046310 (2003).

[15] R. Ledesma-Aguilar, M. Quevedo-Reyes, E. Corvera Poiré, and A. Hernández-Machado, Phys. Rev. E 71, 016312 (2005).

[16] M. Quevedo-Reyes, A. Hernández-Machado, and E. Corvera Poiré, Phys. Rev. E 73, 066308 (2006).

[17] The relation $U=V_{\infty} / \lambda$ is derived from mass conservation in the projected two-dimensional problem. Although the problem is actually three-dimensional, because there is a wetting layer of oil left behind the finger on each glass plate, this fact can be neglected in our experiments. The withdrawal velocities $V_{\infty}$ $=1.42$ and $2.83 \mathrm{~mm} / \mathrm{s}$ correspond to capillary numbers $\mathrm{Ca}$ $=\eta U / \sigma=0.06$ and 0.13 , for which the thicknesses of the wetting layers are only $6 \%$ and $7 \%$ of the gap thickness $b$ [19].

[18] C. W. Park and G. M. Homsy, Phys. Fluids 28, 1583 (1985).

[19] G. Z. P. Tabeling, G. Zocchi, and A. Libchaber, J. Fluid Mech. 177, 67 (1987).cd

[20] R. Pieters and J. S. Langer, Phys. Rev. Lett. 56, 1948 (1986).

[21] A. Dougherty, P. D. Kaplan, and J. P. Gollub, Phys. Rev. Lett. 58, 1652 (1987).

[22] E. Corvera Poiré and J. A. del Río, J. Phys.: Condens. Matter 16, S2055 (2004).

[23] E. Corvera Poiré, M. López de Haro, and J. del Río Portilla, US Patent No. 7,201,224 (10 April 2007).

[24] R. Collepardo-Guevara and E. Corvera Poiré, Phys. Rev. E 76, 026301 (2007). 